solvent correction for the salt of a weak acid has been discussed elsewhere 4 and can be calculated if the amount of carbon dioxide dissolved in the water is known. Applying it to Jeffery and Vogel's figures on the assumption that their water was saturated with carbon dioxide at a partial pressure of 0.00035 atm. gives values far greater than those of MacInnes and Shedlovsky, but the figures obtained in this way are undoubtedly too high, since Jeffery and Vogel's water (like that of the American workers) probably contained saline impurities as well as carbon dioxide. Without precise information on this point the correct extrapolation of Jeffery and Vogel's figures is impossible, but it can safely be concluded that the uncertainty fully covers the discrepancy of $1 \cdot 6$ units between the limiting conductivities derived in the two investigations.

It is to this discrepancy, together with a smaller

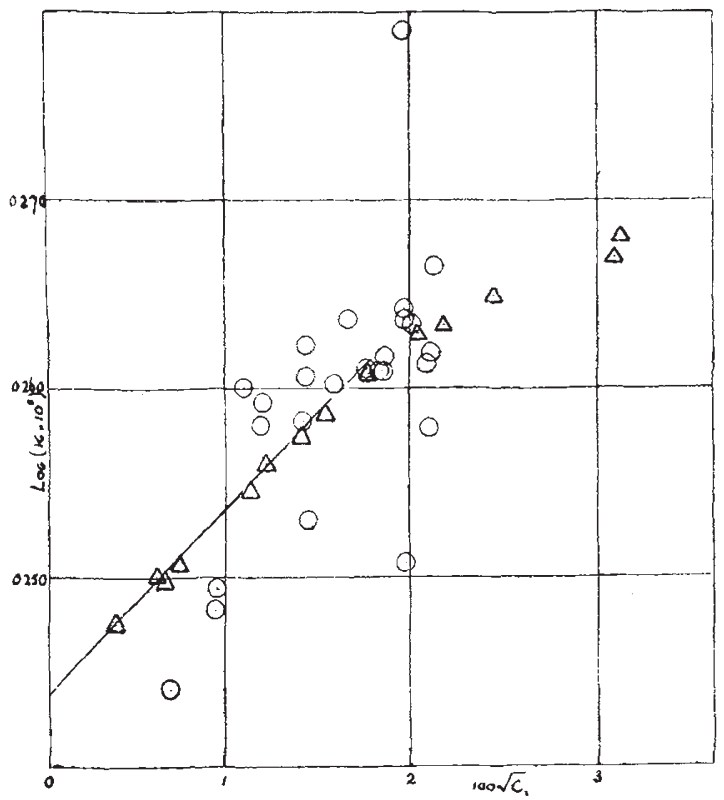

F1G. 1. Dissociation constant of acetic acid. $\bigcirc$, Jeffery and Vogel. $\triangle$, MacInnes and Shedlovsky.

one in the same direction between the values adopted for the mobility of the hydrogen ion, that the differing values reported for the dissociation constant of acetic acid are mainly due; if both series of measurements are compared on a common basis, the same value of $K_{0}$ is obtained. This is shown in Fig. 1, in which the logarithm of the constant, uncorrected for activity changes, is plotted against the square root of the ionic concentration, using for both series the common value $\Lambda_{0}=390 \cdot 59$. The use of this common value neglects the small difference in cell constant bases, which Jeffery and Vogel regard as within the limits of accuracy that they claim for their measurements.

$$
\text { C. W. Davies. }
$$$$
\text { W. H. BANKS. }
$$

Battersea Polytechnic, London, S.W.11. Jan. 17.

1 J. Ampr. Chem. Soc., 54, 1429; 1932.

\section{Artificial Control of Sex in the Progeny of} Mammals

Genetics and cytology prove that the sex of the progeny of mammals depends upon the sex of the spermatozoon fertilising the ovum; the ovum itself is not determined as to sex. The 'female' spermatozoon possesses an $X$-chromosome, the 'male' possesses a $Y$-chromosome (or no sex chromosome at all), while every ovum has an $X$-chromosome. Male-determining and female-determining spermatozoa are mixed irregularly in the sperm. Therefore there is only one way of controlling the sex of progeny in mammals, namely, by separating the male-determining from the female-determining spermatozoa.

Spermatozoa with $X$-and with $Y$-chromosome are derived from one asymmetric mother $(X+Y)$ cell ; it was accordingly natural to suppose that they would carry electrical charges of opposite sign. We accordingly tried to isolate them with an electric current. Most living cells during cataphoresis go to the anode, their surfaces being electrically negative. When we passed an electrical current through horse or rabbit sperm in physiological solution, we found that part of the spermatozoa went to the anode, part to the cathode, while the rest remained some time in the middle in the horizontal part of the Michaelis apparatus.

We have artificially impregnated three female rabbits with three portions of sperm after cataphoresis. The female impregnated with anode spermatozoa produced six young, all of the female sex; the second, impregnated with cathode sperm. atozoa, produced four males and one female; and the third, impregnated with the central fraction left between the two poles of the Michaelis apparatus, bore two males and two females. So far, this is the only experiment we have carried out on artificial impregnation with anode- and cathode-spermatozoa, and we propose to verify it on a larger scale. However, it is most improbable that the results were due only to chance. The only exception to what we expected, was a female born after fertilisation with cathode spermatozoa, which can be explained by the diff. culty of obtaining complete isolation by mechanical means of three varieties of sperm in a Michaelis apparatus.

\section{N. K. KoltzofF}

V. N. SCHRÖDER.

Institute of Experimental Biology and Laboratory of Experimental Cytology, Institute of Animal Breeding,

Moscow.

\section{Capacitance Hygroscopy}

I AM obliged to Dr. Schofield for directing further attention to the subsidiary complications of capacitance measurements'. As E. B. Moullin says :" "We are again and again forced to remember that inductance, capacity, and resistance are attributes possessed simultaneously by all apparatus, and we cannot say that this is an inductance, or that is a capacity with the easy nonchalance of the power engineer". To avoid the accusation of such nonchalance, I used inverted cormmas for 'capacitance' in my article, made such cautionary remarks as the limits of space permitted, and used the word 'hygroscopy' instead of 'hygrometry' in its title.

Good demonstrations of these complications can be obtained by making various series and parallel 